\title{
Point-contact spectroscopy of the normal state excitations in $\mathrm{PrOs}_{4} \mathrm{Sb}_{12}$
}

\author{
O. E. Kvitnitskaya ${ }^{\text {a,* }}$, Yu. G. Naidyuk ${ }^{\text {a }}$, I. K. Yanson ${ }^{\text {a }}$, A. Karkin ${ }^{\text {b }}$, S. Naumov ${ }^{\text {, }}$, \\ N. Kostromitina ${ }^{b}$ \\ ${ }^{\mathrm{a}}$ B. Verkin Institute for Low Temperature Physics and Engineering, NASU, 47 Lenin Ave., 61103, Kharkiv, Ukraine \\ ${ }^{\mathrm{b}}$ Institute of Metal Physics, S. Kovalevskoi Str. 18, Ekaterinburg 620219, Russia
}

\begin{abstract}
Point-contact $(\mathrm{PC})$ investigations of the heavy-fermion $(\mathrm{HF})$ superconductor $\mathrm{PrOs}_{4} \mathrm{Sb}_{12}$ in the temperature range between 1.5 and $4.2 \mathrm{~K}$ and in a magnetic field up to $8 \mathrm{~T}$ are presented. The main feature in PC spectra, the second derivative of the $\mathrm{I}-\mathrm{V}$ curves, in the normal state is a peak at about $1-3 \mathrm{mV}$. This low energy peak is smeared by the temperature rise and suppressed (or splitted) by a magnetic field. The origin of the peak is likely excitation of the $\mathrm{Pr}^{3+}$ ion from the ground $\Gamma_{1}$ state to the upper $\Gamma_{5}$ state, considering the generally accepted crystalline electric field (CEF) schema in $\mathrm{PrOs}_{4} \mathrm{Sb}_{12}$. Absence of the visible features of other CEF transitions in the PC spectra testifies to the dominant coupling of the conducting electrons with this low-lying (about $1 \mathrm{meV}$ in energy) excitation. Thus, this interaction is important for ascertainment of the nature of $\mathrm{HF}$ and superconducting state in $\mathrm{PrOs}_{4} \mathrm{Sb}_{12}$.
\end{abstract}

Key words: $\mathrm{PrOs}_{4} \mathrm{Sb}_{12}$, crystalline electric field, point-contact spectroscopy PACS: 71.70.Ch, 71.70.Ej, 73.40.Jn, 74.70.Tx

$\mathrm{PrOs}_{4} \mathrm{Sb}_{12}$ appears to be the first Pr-based heavyfermion superconductor $\left(\mathrm{T}_{c}=1.85 \mathrm{~K}\right)$ with an effective quasiparticle mass $m^{*} \approx 50 m_{e}$ according to the enhanced electronic specific heat coefficient $\gamma[1]$. Crystalelectric-field (CEF) splitting of the $\operatorname{Pr}^{3+}$ multiplet influences the physical properties of $\mathrm{PrOs}_{4} \mathrm{Sb}_{12}$ as widely accepted, first of all, the heavy-fermion phenomena and superconducting pairing mechanism in this compound. According to the recent specific heat, magnetization, elastic and inelastic neutron scattering measurements [2] CEF schema in $\mathrm{PrOs}_{4} \mathrm{Sb}_{12}$ tends to be as follows: magnetic $\Gamma_{5}$ and $\Gamma_{4}$ triplets, nonmagnetic $\Gamma_{3}$ doublet separated from the ground $\Gamma_{1}$ state by $10 \mathrm{~K}$, $100 \mathrm{~K}$ and $300 \mathrm{~K}$ respectively. Thus, as it is commonly believed, mass enhancement would arise from inelastic

\footnotetext{
* Corresponding author. Tel: $++380-57-3402211$ fax: $++(380)-57-340-33-70$

Email address: kvitnitskaya@ilt.kharkov.ua (O. E. Kvitnitskaya).
}

exchange scattering of the conduction electrons by the well-localized hybridized $4 f^{2}$ electrons of the $\operatorname{Pr}^{3+}$ ion.

Point-contact spectroscopy (PCS) turns to be a successful tool for the direct study of energy-dependent interactions between conducting electrons and different quasiparticle excitations in metals [3]. In [4] a PCS theory for the $f$-localized states was evolved. It was shown that nonlinear conductivity of PC is determined by the inelastic scattering of the conducting electrons on the $f$-shell, what allows to probe the CEF levels of the rare-earth ion. PCS was used successfully to study the CEF levels of the $\mathrm{Pr}^{3+}$ ion in $\mathrm{PrNi}_{5}$ and their Zeeman splitting [5]. In the case of heavy-fermion materials obtaining of the spectroscopic information is restricted by their very high resistivity that leads to violation of the ballistic condition and transition to the thermal regime and dominance of the self-heating effects in the PC spectra. Only PC's on relatively "low resistivity" specimens $(\leq 10 \mu \Omega \mathrm{cm})$ which we believe our $\mathrm{PrOs}_{4} \mathrm{Sb}_{12}$ samples belong to, may have at low 
voltage inelastic electron mean free path exceeding the PC size and spectra with spectroscopic features.

We have used the $\mathrm{PrOs}_{4} \mathrm{Sb}_{12}$ single-crystal samples $\left(\mathrm{T}_{c}=1.75 \mathrm{~K}\right)$ of a sub-millimeter size. To produce $\mathrm{PC}$ 's a $\mathrm{Cu}$ electrode had a gentle touch to 'as grown' $\mathrm{PrOs}_{4} \mathrm{Sb}_{12}$ surface in liquid $\mathrm{He}^{4}$. The first and second derivatives of the $\mathrm{I}-\mathrm{V}$ curves as function of bias voltage were recorded using a standard lock-in technique.

In Fig. 1 the $d V / d I(V)$ characteristics of $\mathrm{PC}$ between $\mathrm{PrOs}_{4} \mathrm{Sb}_{12}$ and $\mathrm{Cu}$ taken at $\mathrm{T}=1.5 \mathrm{~K}$ are presented for the magnetic field range between 0 and $8 \mathrm{~T}$. The 'quasi' parabolic $d V / d I(V)$ curves show also a minimum around $\mathrm{V} \simeq 0 \mathrm{mV}$. Magnetic field somewhat degrades the zero-bias minimum.

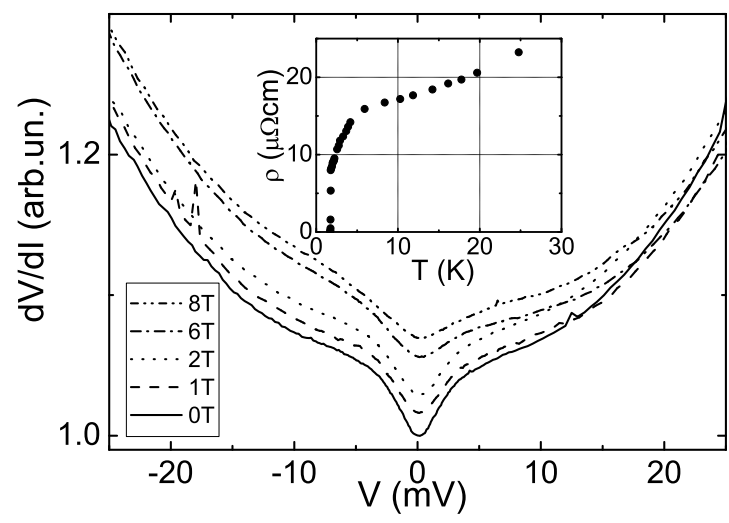

Fig. 1. Experimental differential resistance $(d V / d I)$ of a $\mathrm{PrOs}_{4} \mathrm{Sb}_{12}-\mathrm{Cu}$ PC with $\mathrm{R}_{0}=0.8 \Omega$, in magnetic field at $\mathrm{T}=1.5 \mathrm{~K}$. Inset: specific resistivity of $\mathrm{PrOs}_{4} \mathrm{Sb}_{12}$.

Figure 2 displays PC spectra $d^{2} V / d I^{2}(V)$ corresponding to Fig. 1. Zero magnetic field spectrum shows sharp peak at about $0.8 \mathrm{mV}$, which is suppressed by magnetic field of $0.5 \mathrm{~T}$. Traces of superconductivity are likely responsible for this feature.

The most prominent peculiarity of the spectra is a maximum at about $1.8 \mathrm{mV}$. Increasing temperatures (not shown) as well as magnetic fields lead to its broadening, reduction and finally even splitting is seen at high magnetic fields. This maximum can be connected with the allowed CEF transition $\Gamma_{1} \rightarrow \Gamma_{5}$ with energy about $0.7 \mathrm{meV}$, according to the level scheme determined in [6]. Next allowed CEF transition (around $11 \mathrm{meV}$ [6]) is not displayed in the PC spectra pointing out to the dominant coupling of the conducting electrons with the low-lying $\Gamma_{1} \rightarrow \Gamma_{5}$ excitation. Moreover, no features due to phonon maxima of $\mathrm{Cu}$ (around $16 \mathrm{meV}$ ) as well as $\mathrm{PrOs}_{4} \mathrm{Sb}_{12}$ phonons were enucleated (top inset in Fig. 2). This may be associated with the small contribution from phonon compared to the $\mathrm{CEF}$ excitations and transition of the $\mathrm{PC}$ region in the thermal regime at higher voltages $(>10 \mathrm{mV})$ due to decrease of the inelastic mean free path.

Figure 2 (bottom inset) shows a calculation according to the CEF theory [4] compared to the experimental curve taken at $1 \mathrm{~T}$ to suppress the mentioned $0.8 \mathrm{mV}$ peak. The main parameter of the calculation is the position of the CEF peak taken at $1.1 \mathrm{meV}$ to fit the experimental curve what is in a reasonable agreement with the neutron data [6]. Contrary, calculation of the spectrum in the thermal regime according to Eq.(3.23) in [3] (Fig. 2, upper inset) exhibits a maximum at $0.6 \mathrm{mV}$ with a shallow hump around $20 \mathrm{mV}$.

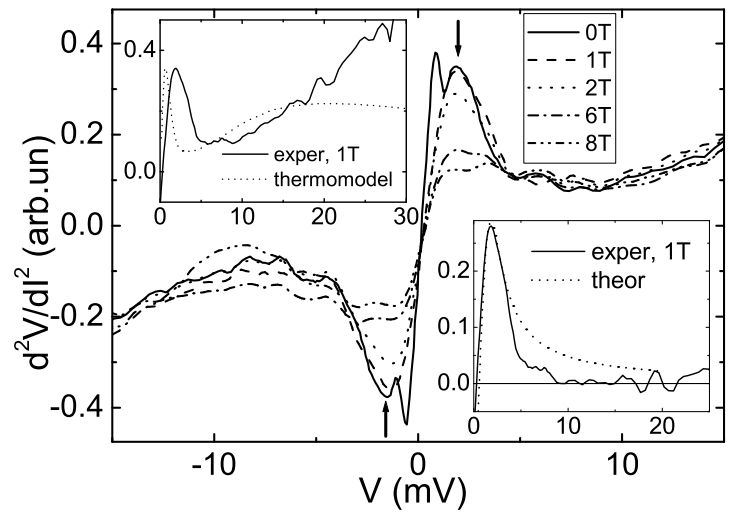

Fig. 2. Experimental spectra $\left(d^{2} V / d I^{2}\right)$ of a $\mathrm{PrOs}_{4} \mathrm{Sb}_{12}-\mathrm{Cu}$ PC from Fig.1. Arrows mark the CEF peak. Top inset: Zero magnetic field $d^{2} V / d I^{2}$ of the same contact at $B=1 \mathrm{~T}$ in the wider bias range in comparison with the thermal regime calculation (see text). Bottom inset: $d^{2} V / d I^{2}$ curve at $1 \mathrm{~T}$ with the subtracted background of the same PC (solid) compared to the theoretical one (dotted) calculated according to the theory [4].

Finally, we have presented the first results of PCS for the skutterudite $\mathrm{PrOs}_{4} \mathrm{Sb}_{12}$. For low bias a pronounced maximum in the PC spectra is well resolved. This maximum is suppressed, broadened and splitted by a magnetic field what points out to its CEF excitation nature. To reinforce this, measurements at lower temperature and higher magnetic field are desirable.

\section{References}

[1] E.D. Bauer, et al., Phys. Rev. B 65 (2002) 100506.

[2] M.B. Maple, J. Phys. Soc. Jpn., 74 (2005) 222.

[3] Yu. G. Naidyuk and I. K. Yanson, Point Contact Spectroscopy, Springer Series in Solid-State Sciences, Vol.145 (Springer, New York, 2004).

[4] I.O. Kulik, et al., Sov. J. Low Temp. Phys. 14 (1988) 82.

[5] M. Reiffers, et al., Phys. Rev. Lett. 62 (1989) 1560. 
[6] E.A. Goremychkin, et al., Phys. Rev. Lett. 93 (2004) 157003. 